\section{Diagnostik und Therapie bei Schwindel}

$\mathrm{D}$ as Lehrbuch gibt zunächst einen umfassenden Überblick über Anatomie und Physiologie des Gleichgewichtssystems sowie über orientierende Untersuchungsmethoden am Krankenbett mit fragebogengestützter Anamneseerhebung und über aufwendige Verfahren zum Nachweis einer Störung des Otolithenund Bogengangsystems. Dadurch unterscheidet es sich nicht von anderen Büchern namhafter deutscher Neurootologen, ist jedoch sehr verständlich auch für Einsteiger geschrieben, mit guten Zeichnungen versehen und durch übersichtliche Merksätze untermauert.
Dem Leser wird die Vielfältigkeit des sechsten Sinnes vor Augen geführt. Es werden nicht nur alle gängigen Krankheitsbilder beschrieben, die zu einem peripheren Schwindel führen, die entsprechenden Untersuchungsmethoden aufgeschlüsselt und therapeutische Möglichkeiten vorgestellt, sondern auch gängige zentral-vestibuläre Störungen erläutert. Darüber hinaus werden durch namhafte Co-Autoren Gleichgewichtsstörungen vorgestellt, die durch neurologische, psychiatrische, internistische (z.B. Synkopen), orthopädische (z.B. HWS-Traumen) oder ophthalmologische (z.B. Abbil-

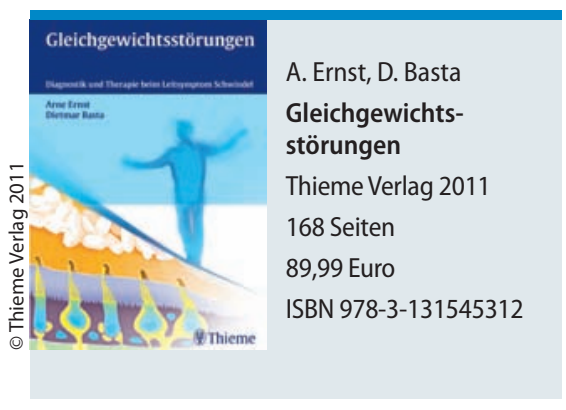

dungsfehler) Krankheiten verursacht werden, auch bei Kindern und alten Menschen. Von den spezifischen Therapien wird besonders auf Rehabilitationstechniken eingegangen, sowie auf die Sturzprophylaxe bei Presbyvertigo.

Prof. Dr. med. Karin Schorn

\title{
Handreichungen zur Stimmdiagnostik
}

$\mathrm{M}$ it dem Untertitel „aus der Praxis für die Praxis“ empfehlen die Herausgeber das Buch, das aus Vorträgen zu einem seit 2006 jährlich gehaltenen Kurs „Stimmdiagnostik“ und den dabei im Austausch mit den Teilnehmern gesammelten Erfahrungen hervorgegangen ist, als ein Kompendium für die praktische Arbeit von HNO-Ärzten, Logopäden und Phoniatern. Sie betonen, dass klinisch-praktische Aspekte den Inhalt bestimmen und nicht primär theoretische Darstellungen, berücksichtigen aber auch, dass ohne entsprechende Grundlagenkenntnisse eine zutreffende klinische Bewertung der erhobenen Befunde nicht erwartet werden kann. Es wird hier also keine simple Anweisung für den Gebrauch eines diagnostischen Werkzeugkastens vorgelegt, sondern eine
Zusammenstellung von Untersuchungsmethoden, deren physikalische bzw. physiologische und auch klinische Bezüge immer in Betracht gezogen sind. Erörtert werden Anamnese, Atmungsuntersuchung, Stimmumfangsprofile, Belastbarkeit, Tauglichkeit (Seidner), Selbstbewertung, Gesamtmaße, Schwingungsanalyse (Nawka), perzeptive Beurteilung (Anders), Kehlkopfuntersuchung (Zorowka), Phonochirurgie (Seidner, Nawka), akustische Verfahren, Besonderheiten bei Kindern und Jugendlichen (Fuchs) und außerdem Diagnostik und Therapieevaluation von Schluckstörungen (Schröter-Morasch). Besonders hervorzuheben ist das Kapitel Dokumentation (Nawka, Seidner), in dem an Hand von 25 Doppelbildern des Larynx Kehlkopfbefunde mit anderen Befunden der

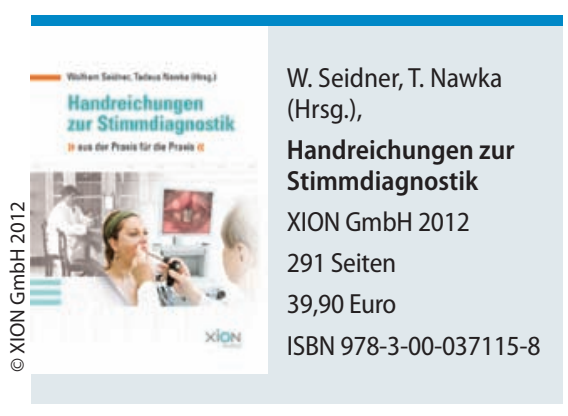

Stimmdiagnostik präsentiert werden und im Zusammenhang mit therapeutischen Konsequenzen sehr einprägsam zur Darstellung kommen. Alles in allem eine von kompetenten Fachleuten zusammengestellte, klar strukturierte, auf das Wesentliche konzentrierte Fibel mit exzellenten Abbildungen - aus der Praxis für die Praxis uneingeschränkt $\mathrm{zu}$ empfehlen

Prof Dr. med. Jürgen Wendler

\section{Leitfaden für die ärztliche Praxis}

omplementärmedizinische Verfahren sind bei den Patienten beliebt und werden auch für Schulmediziner zunehmend interessanter.

In einem neuen Leitfaden hat ein $\mathrm{Au}$ torenteam die verschiedenen Methoden der Naturheilkunde ausführlich behandelt. Neben den evidenzbasierten klassischen Methoden wie Hydro- und Ther- motherapie, Bewegungstherapie oder Phytotherapie werden auch die Homöopathie und die anthroposophische Medizin dargestellt.

Eine gute Übersicht bietet die Darstellung der verschiedenen Indikationsbereiche mit den Behandlungsmöglichkeiten, die die verschiedenen Therapierichtungen bieten.

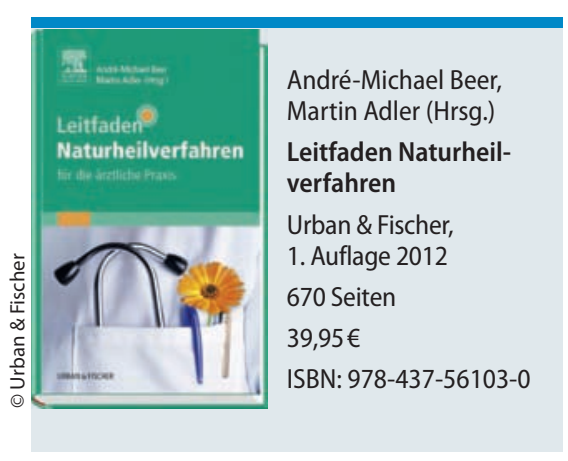

